\title{
Case Report: Asymptomatic Mediastinal Tumor: Role of Preventive Screening in Early Detection and Survival Forecast
}

\author{
Zeynapur Aida Ali-Agaevna ${ }^{1,}$, , Drapkina Oxana Mikhailovna ${ }^{1}$, Shutov Sergei Alexandrovich ${ }^{2}$, \\ Kovrigina Alla Mikhailovna ${ }^{2}$ \\ ${ }^{1}$ Federal Medical Establishment "National Medical Scientific and Research Center of Therapy and Preventive Medicine" of the Ministry of \\ Health of the Russian Federation, Moscow, Russia \\ ${ }^{2}$ Federal Medical Establishment "National Medical Research Center for Hematology" of the Ministry of Health of the Russian Federation, \\ Moscow, Russia
}

Email address:

AZeynapur@gnicpm.ru (Z. A. Ali-Agaevna)

${ }^{*}$ Corresponding author

\section{To cite this article:}

Zeynapur Aida Ali-Agaevna, Drapkina Oxana Mikhailovna, Shutov Sergei Alexandrovich, Kovrigina Alla Mikhailovna. Case Report: Asymptomatic Mediastinal Tumor: Role of Preventive Screening in Early Detection and Survival Forecast. American Journal of Health Research. Vol. 8, No. 5, 2020, pp. 78-83. doi: 10.11648/j.ajhr.20200805.11

Received: September 9, 2020; Accepted: October 9, 2020; Published: October 23, 2020

\begin{abstract}
The main presentation is to use the opportunities and advantages of the annual dispensary examination. Screening of the population allows early detection of the disease associated with reduced working capacity, disability and high mortality among the adult population. Later diagnosis at the stage of advanced manifestations or complications worsens the prognosis and survival. Currently, according to the World Health Organization (WHO), diseases of the cardiovascular system, cancer and diabetes mellitus, along with infectious diseases, contribute to the main morbidity and mortality worldwide. The preventive medicine strategy allows, on the one hand, to have a positive effect on the health of the population and overall life expectancy, on the other hand, to significantly reduce the financial burden on the entire health care system. At the same time, tumor lesions of mediastinum are mostly asymptomatic until they spread beyond the capsule and grow into neighboring vital organs - the heart, aorta, pulmonary arteries, lungs and esophagus. In this clinical case report, the use of the method of regular annual preventive examinations (complete blood count, urine, electrocardiography, chest X-ray, etc.) in this clinical case made it possible to identify tumor lesions of the anterior mediastinum at the stage of preclinical or early clinical manifestations leading to disability and mortality. As a result of the timely provision of medical care based on the latest recommendations of the national specialized communities, the total removal of the tumor formation made it possible not only to improve the prognosis, but also to achieve complete recovery.
\end{abstract}

Keywords: Thymoma, Ophthalmoplegic Myasthenia Gravis, Horner's Syndrome, Thoracoscopic Resection of Mediastinal Tumors

\section{Introduction}

Any disease, regardless of the nature and localization of the pathological process, goes through several stages of development. This is the stage of initiation (preclinical period), the stage of clinical manifestations with varying degrees of severity of symptoms and the final stage, which can take the form of complete recovery, residual functional disorders with varying likelihood of relapse (stage of remission), or the death of the patient with unsuccessful treatment. Several critical factors directly affect survival, including, but not limited to, the timeliness and accuracy of treatment, and patient compliance with all medical, diagnostic, and preventive measures.

The peculiarity of tumors of the thymus is that they belong to the group of rare tumors, rare among all types of cancer.. So is the long asymptomatic period of the course. By the time of the appearance of clinical manifestations, the tumor, as a 
rule, is significantly increasing, spreads to neighboring vital organs. In this situation, the first stage is early diagnosis, the accuracy of which determines tactics and affects prognosis and survival. However, diagnostic search, in turn, begins after the appearance of complaints, forcing visits to doctors. This means that an appeal to a medical institution occurs at the stage of clinical manifestations. In recent decades, the Russian Federation has been actively implementing annual preventive examinations in order to both assess the health status of targeted population and identify risk factors for the development of non-communicable diseases as well as to identify diseases at the preclinical stage.

In this case, treatment, started before the onset of irreversible complications, can not only save lives, but also achieve better results at lower costs and without the compulsory use of techniques with significant side effects that could aggravate the course of the disease, while imposing a financial burden on the health care system. In this context, the main objective of this article is to present the case of a patient with a largely asymptomatic development of the mediastinum tumor, the identification of which was made possible by an annual preventive examination, particularly through a blood test and following PET-CT.

\section{Case Presentation}

We would like to present a clinical case of a 54-year-old woman, who leads an active lifestyle with significant physical and psychosocial overloads that did not previously limit her activity. During the annual clinical examination with a chest x-ray, complete blood count, urine, mammography, examination by a gynecologist and therapist, no abnormalities were detected. A several months before the next medical examination, the patient noted rare episodes of unexpressed ptosis of the upper eyelid of the left eye that appeared briefly in the second half of the day (Figure 1).

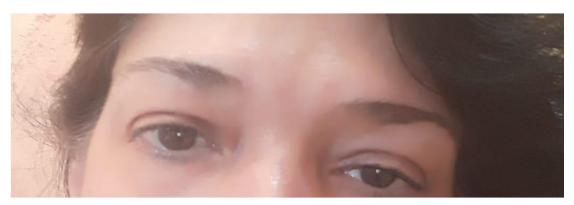

Figure 1. Upper Eyelid Ptosis of the Left Eye.

The rarity, short duration and lack of expression of ptosis without significant visual impairment did not worsen the quality of life. No pathology was revealed by an ophthalmologist and a neurologist during examination outside of transient ptosis, and no further examination was carried out. Some time later, the patient started noticing fatigue and the appearance of shortness of breath with significant exertion. Amid increasing in frequency visual disturbances, the next clinical examination identified a significant increase in the level of lymphocytes - both in relative and in absolute terms -- in the patient's complete blood test. Comparing the changes in blood tests results with the clinical picture of transient ptosis of the eyelid, a lymphoproliferative disease with mediastinal lesion with
Horner's syndrome was suspected. In this context, a more detailed blood test was conducted with the determination of antibodies to acetylcholine receptors. The test turned negative, which made it possible to exclude the diagnosis of Myasthenia gravis, suggesting secondary myasthenic syndrome against the background of a primary mediastinal lesion. Positron emission tomography, combined with a computed tomography with the introduction of Ffluorodeoxyglucose (18F-FDG PET-CT), revealed moderate hepatomegaly and a volumetric formation of the anteriorsuperior mediastinum, most consistent with lymphoma; no lymph node was detected (Figure 2).

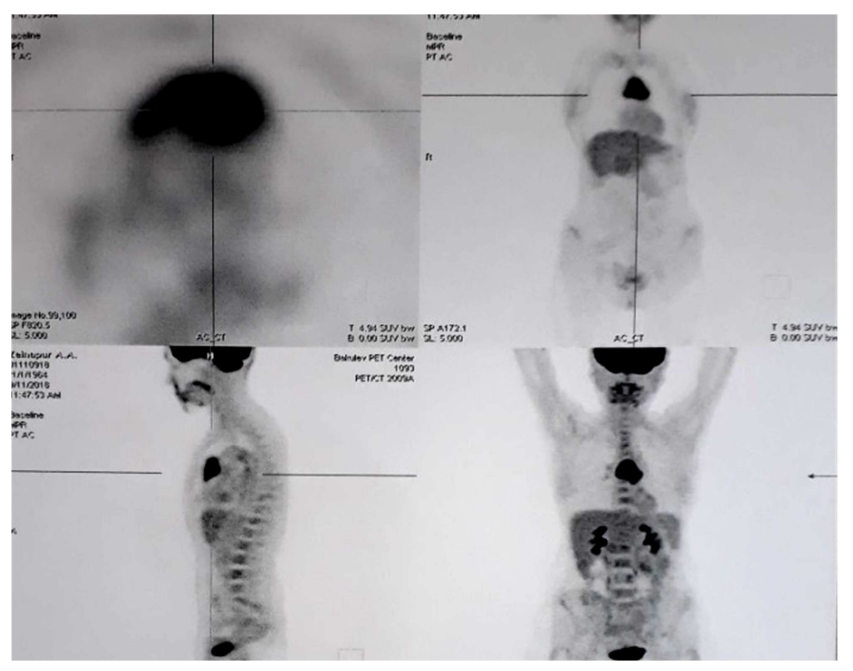

Figure 2. 18F-FDG PET-CT Scans.

Based on the examination results, a preliminary diagnosis was of tumor of the anterior-superior mediastinum with several possibilities: primary mediastinal (thymic) large Bcell lymphoma thymoma Ophthalmoplegic myasthenic syndrome.

The patient was admitted to the hospital and a transthoracic echocardiography was performed. It identified a formation of the anterior-superior mediastinum of a homogeneous structure with a dense capsule; constriction and lumen between the formation and the anterior wall of the ascending aorta, the initial sections of the brachiocephalic trunk, the pulmonary artery trunk and the right atrial appendage; unexpressed dilatation of the right atrium; undisturbed myocardial contractility; and no signs of hypertrophy or valvular pathology. A video-assisted thoracoscopy with the left infusion revealed a mobile dense neoplasm of the anterior superior mediastinum with the dimensions of $60 \times 70 \times 25 \mathrm{~mm}$ (Figure 3a). Resection of $1 \backslash 2$ part of the tumor was performed. According to the emergency intraoperative analysis of the mediastinal tumor biopsy according to rearrangements of genes of the heavy chain of immunoglobulins, B-cell clonality was not revealed. An urgent postmortem examination indicated type B1 thymoma. It was henceforth decided to perform a radical removal of the mediastinal tumor within one intervention. Thoracoscopic dissection of the right pleural cavity was performed with the removal of the rest of the tumor along 
with capsule (Figure 3b).

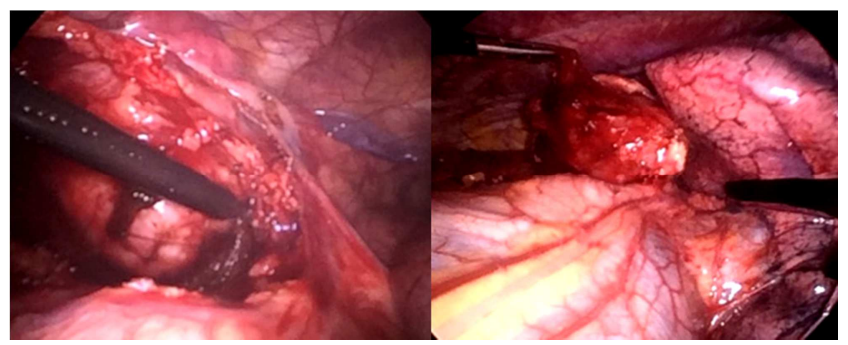

Figure 3. $3 a$ (Left). Anterior Superior Mediastinum During Video-assisted Thoracoscopy.; and $3 b$ Operation of Radical Thoracoscopic Tumor Removal

According to the data of the final intravital pathoanatomical study, tissue sections of an encapsulated neoplasm of a multi-lobular structure, caused by fibrous septa, could be described as follows. The structure was twocomponent: a prevailing component of small lymphoid cells with the presence of mitotic figures, resembling a "starry sky", and a morphologically indistinct epithelial component of small cells with rounded nuclei. Foci of trans- and pericapsular propagation were noted. Some of the sections contained preexisting tissue of the normal thymus with evolutive changes. The obtained pathomorphological data corresponded to type B1 thymoma with trans- and pericapsular growth. In this context, the patient was discharged on the $4^{\text {th }}$ day in a stable condition with the diagnosis of a malignant formation of the thymus gland: thymoma T1N0M0, type BI according to Masaoka-Koga.

Post-surgery blood tests revealed the normalization of the level of lymphocytes. Control 18F-FDG PET-CT of the chest six months following the surgery didn't indicate the presence of extra-nodal foci of pathological accumulation (Figure 4).

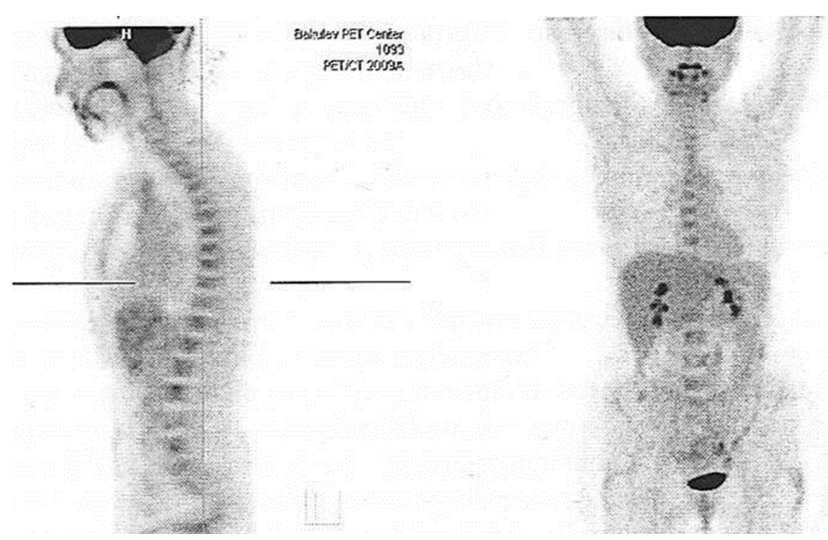

Figure 4. Post-surgery PET-CT Scan.

\section{Discussion}

Currently, according to the World Health Organization (WHO), diseases of the cardiovascular system, cancer and diabetes mellitus, along with infectious diseases, make the main contribution to morbidity and mortality worldwide [1]. At the same time, tumor lesions of the mediastinum are mostly asymptomatic until they spread outside the capsule and germinate into neighboring vital organs - the heart, aorta, pulmonary arteries, esophagus $[2,3]$.

In the presented clinical case, the only complaint of the patient was transient unilateral ptosis of the upper eyelid of the left eye as well as lymphocytosis revealed during the dispensary examination in the general blood test. Taking into account complaints of ptosis, a differential diagnosis was made between diseases associated with Horner's syndrome and ophthalmoplegic myasthenia gravis.

In 1896 a Swiss ophthalmologist from the University of Zurich Johann Friedrich Horner first described ptosis of the upper eyelid, resulting from a violation of the sympathetic innervation of the upper tarsal muscle [4]. Subsequently, the oculosympathetic paresis in the literature began to be referred to as Horner's syndrome. The cause of this syndrome is a violation of innervation due to damage both at the level of the first neuron (hypothalamus-ciliospinal center); and in the lower parts of the second (preganglionic) and third (postganglionic) neurons. The presence of bulbar symptoms, manifestations of plexopathy, and distal neuropathy make it possible to accurately determine the zone of the pathological focus. At the same time, Horner's syndrome, in addition to ptosis, results in other unilateral disorders associated with sympathetic dysfunction on the ipsilateral side - miosis, enophthalmos, anhidrosis, and impaired pupil photoreaction. Given the absence of the above-described symptoms, the oculosympathetic syndrome and associated diseases were excluded from the diagnostic search.

Another cause of eyelid ptosis may be Myasthenia gravis (MG), which is manifested by muscle weakness due to impaired neuromuscular transmission. One of the forms of this autoimmune disease is myasthenia gravis ophthalmoplegic. In this case, ptosis of the eyelids can be both bilateral and unilateral; is transient, occurs episodically and more often in the afternoon. For a long time, apart from drooping of the eyelid, as a rule, there are no other clinical manifestations of myasthenia gravis. One of the reasons for the development of myasthenic syndrome is considered to be a dysfunction of the thymus gland (thymus). According to the literature, there is a certain correlation between $\mathrm{MG}$ and tumors of the thymus gland, with 10 to 20 percent of patients with myasthenia gravis having thymomas, while 25-45 percent of patients with thymoma have myasthenic syndrome of varying severity [2]. According to Filosso P. L et al, there is a direct relationship between the progression of the growth of thymus formation with the concentration of produced auto-aggressive T-lymphocytes and the appearance of lymphocytosis in the peripheral blood [5]. Thymic hyperplasia leads to excess production of the hormone thymine. This, in turn, triggers a cascade of autoimmune reactions that disrupt the function of muscle receptors due to the overproduction of antibodies to the nicotinic acetylcholine receptor directed against various components of the neuromuscular synapse. Antibodies to the acetycholine receptor are found in 80-90 percent of patients with generalized myasthenia gravis, 50-70 percent of patients with ocular myasthenia gravis and are not detected in healthy people. Due to its high sensitivity and specificity, this study 
is the main laboratory method for confirming myasthenia gravis, although in 15 percent of patients with myasthenia gravis, the test may be negative, the so-called seronegative form of myasthenia gravis $[2,6,13,14]$. Accordingly, in case of any suspicion of myasthenia gravis, one of the mandatory diagnostic methods is multispiral computed tomography of the cut chest to assess the state of the thymus [2].

The second clinical symptom identified in the patient was lymphocytosis in the general blood test without signs of a focal and generalized inflammatory process. The lymphocytic link provides the body's immune defense in response to a viral or bacterial infection. Another reason for an increase in the number of lymphocytes may be a disease of the hematopoietic organs or an autoimmune process. Taking into account the asymptomatic changes in laboratory parameters in the diagnostic plan, it was impossible to exclude the debut of lymphoma or lymphogranulomatosis. The combination of ptosis of the eyelid and an increase in the level of lymphocytes became the basis for differentiating between lymphoproliferative disease and thymic pathology.

Thus, despite the paucity of the clinical picture, the main method allowing to narrow the diagnostic search was MSCT or PET-CT not only to clarify the extent of the lesion, but also to clarify the involvement of the peripheral link of the lymphatic system. According to World Health Organization (WHO) recommendations, computed tomography and / or positron emission tomography / computed tomography are considered mandatory as diagnostic examinations. The most informative method for assessing the prevalence of lymphoproliferation is two-photon positron emission computed tomography with the introduction of Ffluorodeoxyglucose (18F-FDG PET-CT) [2, 7]. This technique allows, with a high degree of specificity and sensitivity, to determine the focus of pathological activity, the prevalence and volume of the affected tissue, and today it is standard for the initial examination in case of suspected lymphoma. According to the 2014 the Lugano classification, this procedure is optimal for patients with a local focus to identify additional zones of metabolic activity, which has a direct impact on the choice of further management tactics [9]. The performed study confirmed the correctness of the diagnostic search, revealing an isolated formation of the anterior-superior mediastinum without affecting the lymph nodes and without other foci of pathological accumulation of the radiopharmaceutical.

In the area of the anterior-superior mediastinum, the following types of volumetric lesions are most common thymomas, lymphomas, extragonadotropic germ cell mesenchial tumors (teratomas, teratoblastomas) as well as possibly a retrosternal growing thyroid gland. Due to the anatomical and physiological features of the mediastinum, masses are clinically asymptomatic for a long time and are detected either during X-ray studies of the chest or at a late stage, when the neurovascular structures of the mediastinum are involved in the pathological process [2]. With the progression of the growth of the neoplasm, the blade of myasthenia gravis, Horner's syndrome is attached.
The clinical picture made it possible to differentiate between mediastinal lymphoma and thymoma proper. The main method that allows not only to make the final diagnosis, but also to determine further tactics, is diagnostic thoracoscopy with video-assisted intraoperative biopsy of the formation. Intraoperative immunohistochemical analysis confirmed type B1 thymoma. Urgent histological examination made it possible to simultaneously perform total removal of the tumor within the healthy tissue during a single intervention.

Thymus masses are rare mediastinal tumors. In Europe they occur with a frequency of 1.7 cases per million per year [9]. In the USA according to statistical analysis the overall incidence of the thymus tumor is 0.13 cases per 100,00 person-years [3]. A feature of tumors of the thymus gland is a long-term period of asymptomatic course, which is why the formation is often detected at a late stage of development. The malignancy of thymic formations directly depends on the degree of invasion and the presence of metastasis, which determines both the management tactics and the long-term prognosis. One of the distinguishing features of thymic tumors is their combination with myasthenic syndrome. The Myasthenia gravis clinic is often the first signal that initiates an assessment of the state of the thymus gland. At the same time, there is a connection between the blocking of muscle acetylcholine receptors by autoantibodies with lymphoid hyperplasia of thymic tumors, as well as the reduction in most cases of myasthenic syndrome after radical thymectomy. According to the degree of infiltration, thymomas are divided into three types - encapsulated, minimally invasive, and invasive. In the first case, the tumor is completely surrounded by a fibrous capsule of varying thickness without signs of infiltration of the entire wall. In the second case, the capsule is infiltrated by the tumor throughout its entire thickness, which is revealed only microscopically. In the third form, the invasion spreads to the surrounding structures - the pericardium, large vessels, lung, pleura, including those with metastases to both regional and distal lymph nodes.

In the analyzed case, considering the intimate adherence of the tumor to the wall of the right atrium, the ascending aorta, the initial sections of the brachiocephalic trunk, the pulmonary artery trunk as well as the absence of changes in blood analyses and radiography according to the data of the previous dispensary examination, the patient had a rapidly progressive growth of tumor formation in the mediastinum without signs of metastasis.

In 1999, the WHO published a classification of thymomas, dividing tumors by histological type into six groups depending on the number of thymic epithelial cells and the proportion of lymphocytes in the formation: $\mathrm{A}, \mathrm{AB}, \mathrm{B} 1, \mathrm{~B} 2$, B3 and C. In type A tumors, malignant cells have a spindleshaped or oval in shape, while in type B tumors, malignant cells are similar to epithelial or dendritic cells. Types B1, B2, and B3 correspond mainly to cortical, cortical thymoma and highly differentiated thymic cancer. Type $\mathrm{C}$ tumors correspond to thymic cancer. Subsequently, a modification was carried out with the addition of the division of thymoma 
according to the histological type according to Masaoka Koga into the classification [10] According to her, thymomas are divided into 4 stages, depending on the invasion of the capsule and adjacent organs (I, II, III), large vessels (IIIa and IIIb), and distant metastases (IVa pleura, pericardium) and IVb (lymph nodes). In 2015, the WHO introduced amendments to the TNM classification of thymus tumors [2, 11]. Thus, all tumors of the thymus, depending on the stage of development and histology, are divided into ABC types IIV according to Masaoka - Koga and TNM stages.

Currently, surgical treatment is the only effectively proven method of treating thymoma, reaching 90-100 percent survival rate in the long-term prognosis for thymomas $\mathrm{A}, \mathrm{AB}$ at stage I - II [2, 12, 15]. In more advanced stages, B2 and B3 thymomas and thymus carcinoma, the survival rate and five-year prognosis are lower. In this case, type B1 thymoma occupied an intermediate position, the prognosis of which directly depends on the completeness of the performed intervention with the removal of the entire tumor with a capsule within the healthy tissue. The role of radiation therapy before and after the intervention, as well as chemotherapy, is warranted at III-IV stages, depending on the presence of invasion into neighboring organs of the mediastinum and the detection of distant metastases [2, 9, 14].

A feature of thymic tumors is the likelihood of recurrence with the appearance of formations in ectopic tissue outside the mediastinum, including in the late period. In this regard, it is mandatory to carry out regular MSCT examinations initially after six and 12 months, then once a year for at least five years.

\section{Conclusion}

Thymus tumors are classified as rare diseases, accounting for 0.2 to $1.5 \%$ of all malignant tumors. Among all types of lesions of the anterior mediastinum, thymoma and carcinoma of the thymus gland are among the most common tumors [2]. According to the National Cancer Institute for Surveillance, Epidemiology and End Results (SEER), the overall incidence of thymoma in the USA is 0.13 cases per 100,000 personyears [3]. For the period 2000-2007, the European crude incidence rate of malignant epithelial tumours of thymus was 1.7 per million per year. Most epithelial thymic cancers are malignant thymoma (rate $=1.4$ ); with age-adjusted annual incidence rates per million, ranging from 0,9 to 2,3.[9]. The presented clinical case demonstrates the effectiveness of preventive screening examination in the early detection of a rare oncological pathology. This is especially important in the 50 -year-old + age group or in groups at high risk of developing cancer, cardiovascular disease and diabetes, death from which could be preventable. However, according to annual statistics from the WHO, they account for the bulk of the total number of cases and deaths in the world [1]. Moreover, this trend applies to all countries - both with low and high income per capita. Early diagnosis based on a minimum volume of standard routine examination allows for an early treatment with a lower risk of adverse effects and a better long-term prognosis, reduces the risk of premature mortality, as well as the financial burden on public health systems in general.

\section{Author Contributions}

Zeynapur A. A. - the patient's attending physician and main author; Drapkina O. M. - supervisor, who assisted in the research; Shutov S. A. - surgeon, who performed a surgical intervention with the removal of tumor formation; Kovrigina A. M. conducted immunohistochemical and pathomorphological research.

\section{Funding}

There is no funding source for this case report.

\section{Conflicts of Interest}

The authors declare no competing conflict interests.

\section{References}

[1] World health statistics 2019: monitoring health for the SDGs, sustainable development goals. Geneva: World Health Organization; 2019. Licence: CC BY-NC-SA 3.0 IGO.

[2] Thymoma and Thymic Carcinoma Treatment (Adult) (PDQ $\left.{ }^{\circledR}\right)$ Health Professional VersionPDQ Adult Treatment Editorial Board. In: PDQ Cancer Information Summaries [Internet]. Bethesda (MD): National Cancer Institute (US); 2002-.2020 Sep 23. PMID: 26389476 NBK66041.

[3] Howlader N, Noone AM, Krapcho M, Miller D, Brest A, Yu M, Ruhl J, Tatalovich Z, Mariotto A, Lewis DR, Chen HS, Feuer EJ, Cronin KA (eds). SEER Cancer Statistics Review, 1975-2017, National Cancer Institute. Bethesda, MD, https://seer.cancer.gov/csr/1975_2017/, based on November 2019 SEER data submission, posted to the SEER web site, April 2020.

[4] J. F. Horner: Über eine Form von Ptosis. In: Klinische Monatsblätter für Augenheilkunde, Stuttgart, 1869, Band 7, S: 193-198.

[5] Filosso, P. L., Venuta, F., Oliaro, A., Ruffini, E., Rendina, E. A., Margaritora, S., Casadio, C., Terzi, A., Rena, O., Lococo, F., \& Guerrera, F. Thymoma and inter-relationshis between clinical variables: A multicentre study in 537 patients. Eur J Cardiothorac Surg. 2014 Jun; 45 (6): 1020-7. doi: 10.1093/ejcts/ezt567.

[6] Matthew Meriggioli, Donald B Sanders. Muscle Autoantibodies In Myasthenia Gravis: beyond diagnosis? Expert Rev Clin Immunol. 2012 Jul; 8 (5): 427-38. doi: 10.1586/eci.12.34.

[7] Iwaya Y, Tekenaka K, Akamatsu T., et al. Primary gastric diffuse large B-cell lymphoma with orbital involvement: diagnostic usefulness of $18 \mathrm{~F}$-fluorodeoxyglucose positron emission tomography. Intern Med. 2011; 50 (18): 1953-6. doi: 10.2169/internalmedicine.50.5524. 
[8] Cheson BD, Fisher RI, Barrington SF, et al. Recommendations for initial evaluation, staging, and response assessment of Hodgkin and non-Hodgkin Lymphoma: the Lugano classification. J Clin Oncol. 2014; 32 (27): 3059-67. doi: 10.1200/JCO.2013.54.8800.

[9] Marta Scorsetti, Francesco Leo, Annalisa Trama, et al. Thymoma and thymic carcinomas. Crit Rev Oncol Hematol. 2016 Mar; 99: 332-50. doi: 10.1016/j.critrevonc.2016.01.012.

[10] A Masaoka, Y Monden, K Nakahara, T Tanioka. Follow-up study of thymomas with special reference to their clinical stages. Cancer. 1981 Dec 1; 48 (11): 2485-92. doi: 10.1002/1097-0142(19811201)48:11<2485::aidcncr2820481123>3.0.co;2-r.

[11] Alexander Marx, John K. C. Chan, Jean-Michel Coindre, Frank Detterbeck, Nicolas Girard, Nancy L. Harris, Elaine S. Jaffe, Michael O. Kurrer, Edith M. Marom, Andre L. Moreira, Kiyoshi Mukai, Attilio Orazi, Philipp Ströbel. The 2015 World Health Organization Classification of Tumors of the Thymus: Continuity and Changes. Journal of Thoracic Oncology Volume 10, Issue 10, October 2015, Pages 1383-1395. https://doi.org/10.1097/JTO.0000000000000654.
[12] Gu Z, Fu J, Shen Y, Wei Y, Tan L, Zhang P, Han Y, Chen C, Zhang R, Li Y, Chen K, Chen H, Liu Y, Cui Y, Wang Y, Pang L, Yu Z, Zhou X, Liu Y, Liu Y, Fang W; Members of the Chinese Alliance for Research in Thymomas. Thymectomy versus tumor resection for early-stage thymic malignancies: a Chinese Alliance for Research in Thymomas retrospective database analysis. J Thorac Dis. 2016 Apr; 8 (4): 680-6. doi: 10.21037/jtd.2016.03.16.

[13] Padda SK, Yao X, Antonicelli A, et al. Paraneoplastic syndromes and thymic malignancies: an examination of the International Thymic Malignancy Interest Group retrospective database. J Thorac Oncol 2018; 13: 436-46. Received: 28 March 2018; Accepted: 20 April 2018; Published: 15 May 2018. doi: 10.21037/med.2018.04.06.

[14] Berghmans T, Durieux V, Holbrechts S, et al. Systemic treatments for thymoma and thymic carcinoma: A systematic review. Lung Cancer. 2018 Dec; 126: 25-31. doi: 10.1016/j.lungcan.2018.10.018.

[15] Comacchio GM, Marulli G, Mammana M, Natale G, Schiavon M, Rea F. Surgical Decision Making: Thymoma and Myasthenia Gravis. Thorac Surg Clin. 2019 May; 29 (2): 203213. doi: $10.1016 /$ j.thorsurg.2018.12.007. 